\title{
POWER PROCESSING UNIT ACTIVITIES IN AIRBUS DS SPACE EQUIPMENTS
}

\author{
Author: Fernando PINTÓ ${ }^{(1)}$ \\ Co-authors: Javier PALENCIA ${ }^{(2)}$, Nicoletta WAGNER ${ }^{(3)}$ Guillaume GLORIEUX $^{(4)}$ \\ (1) Airbus Defence and Space (Airbus DS Crisa), Torres Quevedo 9, 28760 Tres Cantos, Spain, Email: \\ Fernando.Pinto@airbus.com \\ (2) Airbus Defence and Space (Airbus DS Crisa), Torres Quevedo 9, 28760 Tres Cantos, Spain, Email: \\ Javier.Palencia@airbus.com \\ (3) Airbus Defence and Space (Airbus DS GmbH), Claude-Dornier-Str.88090 Immenstaad, Germany, Email: \\ Nicoletta.Wagner@airbus.com \\ (4) Airbus Defence and Space (Airbus DS SAS),Rocade Jean Moulin ,Elancourt France, Email: \\ Guillaume.Glorieux@airbus.com
}

\begin{abstract}
This paper presents the Power Processing Units in Airbus DS Space Equipment.

Electric propulsion has (EP) become a reality in the commercial market, and therefore is not anymore a niche market for earth observation and/or deep space missions. Major players worldwide for Commercial satellite manufacturers are targeting in their current programs, electric satellites solutions for orbit raising (OR) and station keeping (SK) maneuvers. Several EP technologies are competing against each other, in particular Hall Effect Thruster (HET), Gridded Ion Engine (GIE) technology (including Kaufmann and Radio Frequency technologies) and High Efficiency Multistage Plasma (HEMP). The main two parameters used to trade-off the main EP technologies are Thrust/Power ratio and Specific Impulse (ISP). Currently the market is mainly thrust driven, and therefore the dominant technology is HET. HET is the most efficient technology for the Thrust/Power ratio parameter.
\end{abstract}

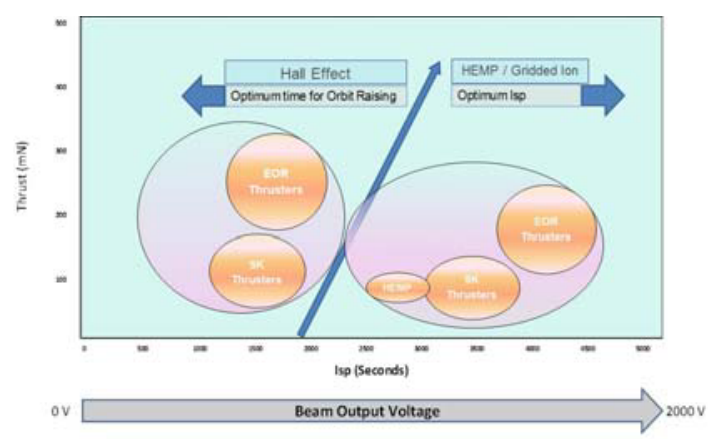

Figure 1. Electric Propulsion Technologies landscape
Airbus DS Space Equipment include in our portfolio PPU solutions for high Thrust ratio and high ISP solutions.

\section{MOTIVATION}

EP business case is driven by current technologies, operator's mindset, and future trends. Several questions with difficult answer can be raised about the future trends for EP. Would the major operators accept OR transfer period above 5 months? How launcher direct injection would impact on the current technologies?

Currently the answers to these questions are not obvious. Airbus DS Space Equipment believes the future is not clear and will most likely not be based on just one single technology, to answer the mid/long term market needs.

In this sense Airbus DS Space Equipment supported by the National Agencies and the European Space Agencies is evolving their current PPU products for high voltage technologies (PPU HV) and developing a new HET PPU solution (PPU Elektro)-able to answer mid-long term market needs.

Airbus DS contains the following PPUs in their portfolio:

$\checkmark$ PPU "Elektro" for HET technology

$\checkmark$ PPU HV for high voltage applications products: GIE and HEMP technologies

$\checkmark$ Generic building blocks to maximize communalities between the different technologies

Figure 2 illustrates the PPU mapping with respect the main EP technologies in the market: 


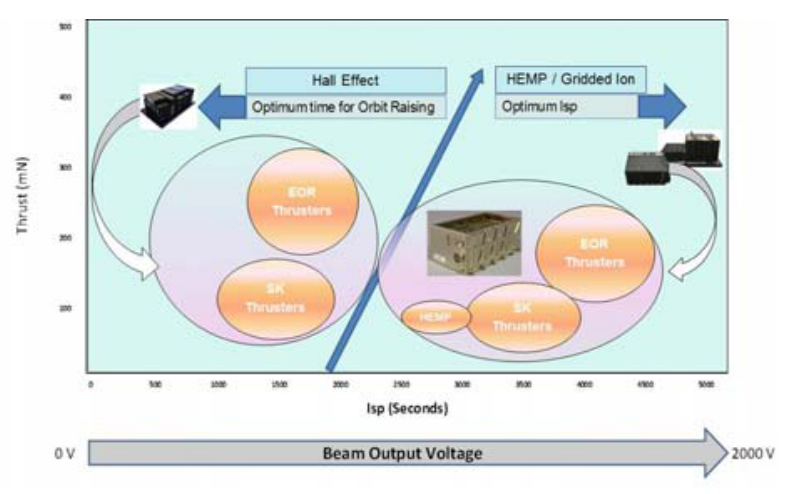

Figure 2 Airbus DS PPU activities to answer to the different Electric Propulsion Technologies

$\checkmark$ PPU "Elektro" product is a joint development between Airbus DS Elancourt (France) and Airbus DS Crisa (Spain). It is a flexible and modular equipment with a new innovative concept of PPU, capable of adapting to a wide range of EP subsystem configurations and compatible to the main HET thrusters in the market. Recently, EM PPU coupling test has been successfully accomplished and the EQM is currently under manufacturing.

$\checkmark$ PPUs for HV applications product. Currently there are already several PPU products available already qualified as follows:

- PPU for T5 is a joint development between Airbus DS Crisa (Spain) and Airbus DS Germany. PPU is a flight proven design in the GOCE mission

- PPU for T6: is a joint development between Airbus DS Crisa (Spain) and Airbus DS Germany. PPU is a qualified design. First mission will be ESA Bepi-Colombo mission. Next step currently under development, is to industrialise existing product in the frame of EGEP program

- PPU for HEMP developed by Airbus DS Germany. PPU is a qualified design and FM already delivered to the customer.

- Next step is to have available the maximum communality between the different technologies. For this-purpose Airbus DS Space Equipment is running a development of generic building blocks to easily customize the PPU to any EP thruster independently of its technology. The maximum communalization will significantly reduce the development efforts, but also the recurrent efforts by applying new technologies and innovative solutions.

Generic building blocks are the basis for the PPU configuration for the different thrusters: PPU for T5
\& T6, PPU for RIT10 and RIT22 and PPU for HEMP. The core element is the generic High Voltage Module, which can be described as a functional block that is designed to be customized to specific thruster needs by exchanging a sub-module without need for a delta-qualification. This modular approach provides the necessary flexibility and maximum of recurring elements. Airbus DS has managed to harmonize these products based on the generic High Voltage Power Supply (HVPS).

\section{PPU ELEKTRO FOR HET}

The PPU Elektro motivation is to respond to Satcom market towards full Electrical Propulsion solutions, capable not only to perform Station Keeping maneuvers, but also Orbit Raising and transfer. Airbus Defence and Space initiated PPU development in order to answer to these needs targeting, but not limited to, $5 \mathrm{~kW}$ class Hall-Effect Thrusters.

This joint development between Airbus DS Elancourt (France) and Airbus DS Crisa (Spain) offers a new innovative concept of PPU, capable to adapt to a wide range of EP subsystem configurations.

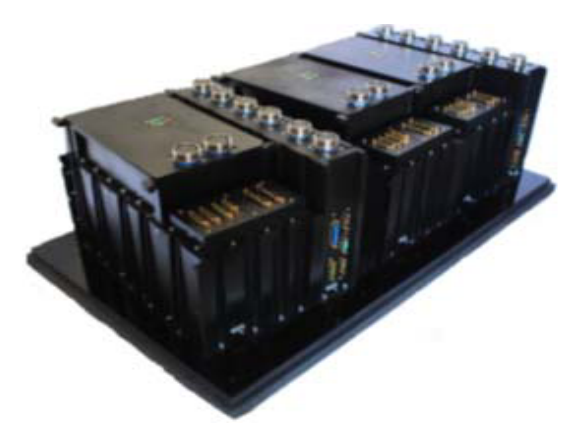

Figure 3 Airbus DS PPU Elektro for HET. Mock-up in $15 \mathrm{~kW}$ configurations

The PPU Elektro offers:

$\checkmark$ An optimised cost solution thanks to an innovative architecture and Design For Manufacturing (DFM) approach based on extensive experience on Telecom Platforms

$\checkmark$ A flexible and modular equipment $\rightarrow$ Same solution able to deliver from $1.5 \mathrm{~kW}$ up to $20 \mathrm{~kW}$. PPU Elektro is thus able to drive up to a max. power of $20 \mathrm{~kW}$ (4 thrusters).

$\checkmark$ The capacity to answer OR and SK needs, with inflight flexibility to configure the unit into a wide range of operating points between 300 to $400 \mathrm{~V}$ (optimize ISP vs thrust)

$\checkmark$ A flexible design compatible to all main HET thrusters in the market.

$\checkmark$ Compatible with a $100 \mathrm{~V}$ power bus (standard for the Telecom Satellites), it could be easily adapted to other power bus voltages. 
Elektro PPU is physically implemented in three main building blocks: Heater-Keeper-Ignitor module (HKI), anode module and Filter thruster switching unit (FTSU) as follows:

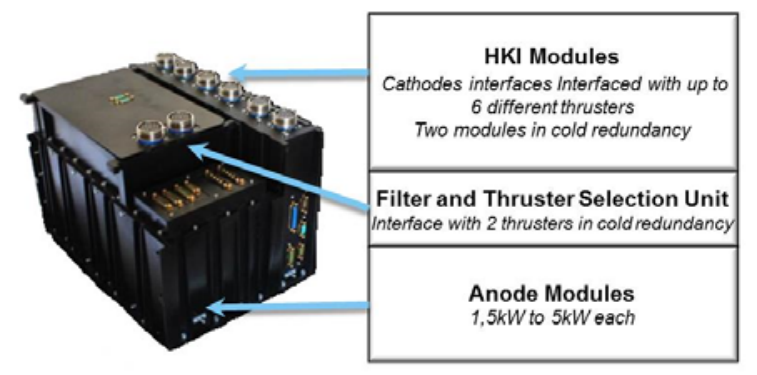

Figure 4 Airbus DS PPU “Elektro” Building Blocks (5 $\mathrm{kW}$ configuration)

The electrical architecture is presented in Figure 5 and 6 The main principle of this architecture is that all functions which are not used permanently in the subsystem (Cathodes Power Supply: Heater, Keeper and Ignitor and sequencing of the overall PPU) are gathered in a module called HKISeq. This module is able to address up to 6 cathodes. All other functions (Anode $5 \mathrm{~kW}$ power supply, Magnet and Xenon Flow Control supplies) are gathered in the module called Anode module. The Filter/Thruster Switching Unit (FTSU) modules are used to filter and switch the Anode power supplies towards 2 thrusters.

Therefore, Elektro PPU architecture and design enables two different configurations of the PPU:

$\checkmark$ Bi-thruster configuration: Able to interface with up to two thrusters

$\checkmark$ Multi-thruster configuration: Up to six cathodes, offering redundancy at PPU level

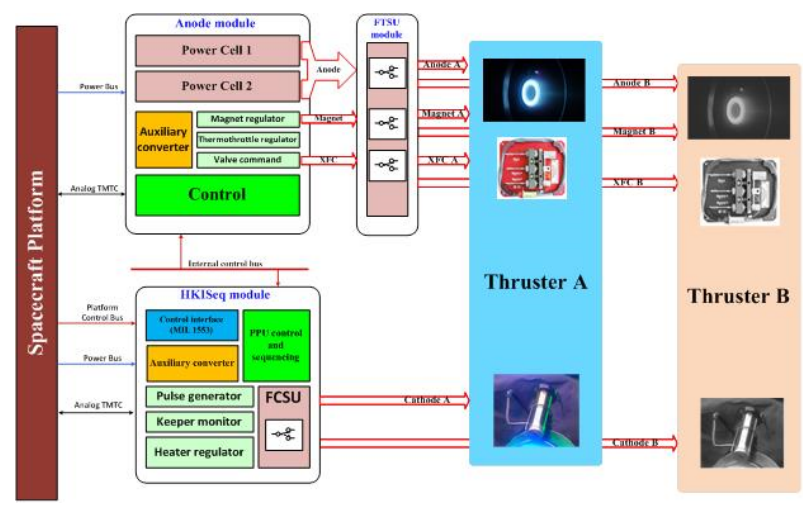

Figure 5 Airbus DS “Elektro” Bi-thruster configuration

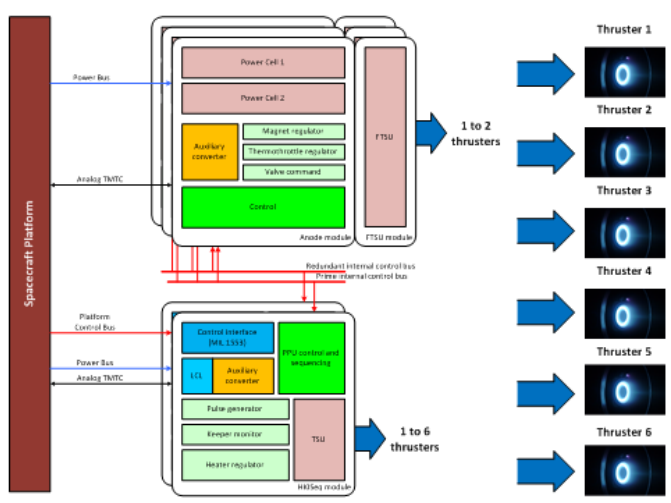

\section{Figure 6 Airbus DS “Elektro” Multi-thruster} configuration

All modules are physically independent and the communication between HKISeq and Anode modules is made by a CAN bus through a harness. Thus, the modules can be assembled altogether with standard fixing points (no specific tie-rods) to form a single unit, or they can be implemented separately.

Examples of the possible configurations are presented in the Figures 3 and 4. This modular approach aims at facilitating accommodation of the PPUs in the spacecraft.

The PPU Elektro development is on-going. The EM PPU already delivered and CDR and EQM manufacturing are currently on going and close to finalisation. The BB and EM model has demonstrated the functionality and performances. The efficiency was measured around $96 \%$ and a characterization of the power limitation has been performed

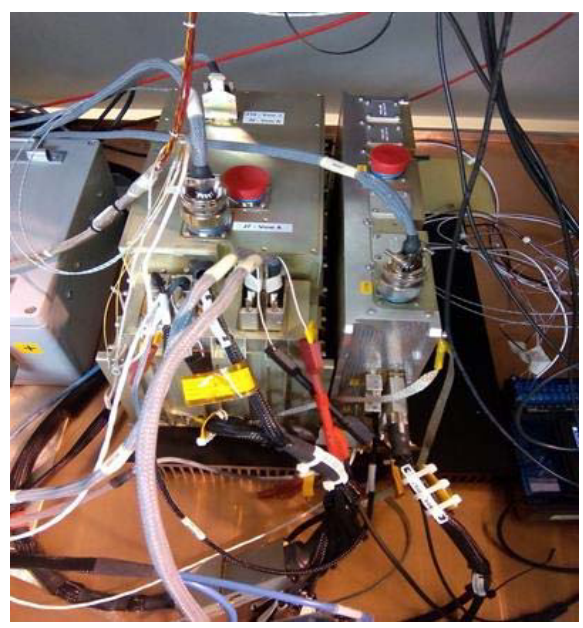

Figure 7 Airbus DS EM PPU Elektro under validation tests in $5 \mathrm{~kW}$ configurations 


\section{HIGH VOLTAGE TECHNOLOGIES PPUS}

\subsection{PPU for T5}

The PPU for QinetiQ T5 thruster, the so called IPCU has been developed by Airbus DS Crisa (Spain) and Airbus DS Germany for the beam output supplies. The IPCU has successfully flown in the ESA GOCE mission.

This unit is meant to be used for low power missions, mainly for earth observation purposes. The main asset of this unit is the high level of controllability from $0.6 \mathrm{mN}$ to $20 \mathrm{mN}$ with a resolution of $12 \mu \mathrm{N}$, with a beam output voltage supply of $1200 \mathrm{~V}$. Refer to Figure 8.

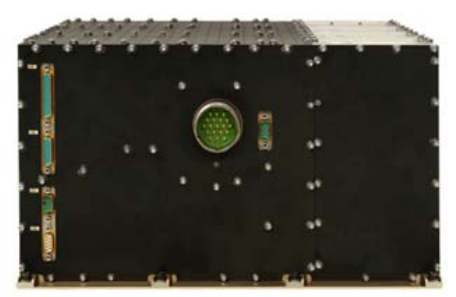

Figure 8 Airbus DS PFM IPCU for T5

\subsection{PPU for T6}

The Power Supply and Conditioning Unit, PSCU, has been developed as a parallel contract to AlphaBus (ESA under ARTES Program). The PSCU has been developed by Airbus DS Crisa and Airbus DS Germany for 1850 Vdc Beam Supply Modules

This unit is the first $5 \mathrm{~kW}$ PPU ever developed in Europe, able to answer to the new full electric satellites needs for the Telecom market and the most demanding deep space missions.

The first flight mission for the PSCU will be ESA BepiColombo mission to Mercury.

The PSCU (see Figure 9) is composed of two Accelerator-Neutralizer Supplies (DANS), housed in single box together with the Beam Supply (BS).

The PSCU T6 design has been upgraded for BepiColombo in order to provide full redundancy and flexibility at PPU level:

$\checkmark$ PSCU T6 enables the system to interface with up to four thrusters, operating one at a time with a total power of $5 \mathrm{~kW}$

$\checkmark$ PSCU T6 is able to offer full redundancy for each of the power supplies

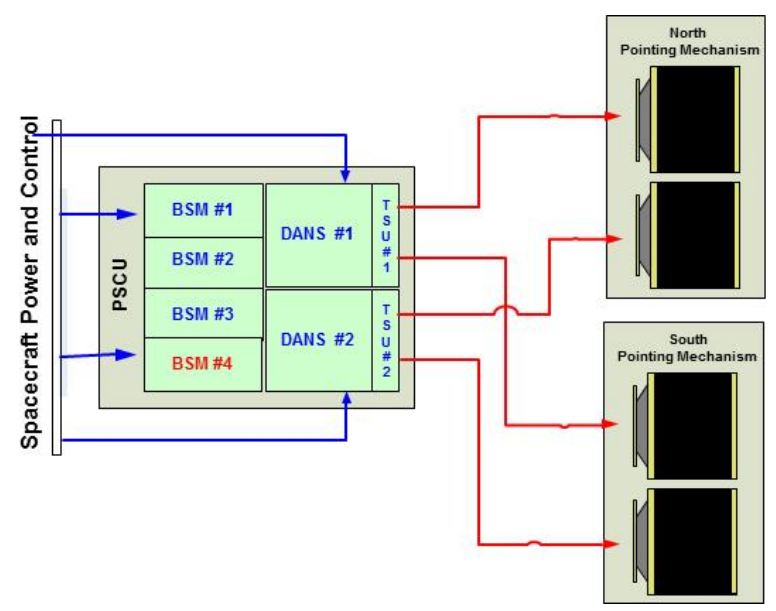

Figure 9 Airbus DS EQM PSCU Architecture

The BEPI PPU EQM has been successfully coupled with the thruster and fully qualified. The flight models (2 FMs) are manufactured and delivered to end customer to support the first flight mission.

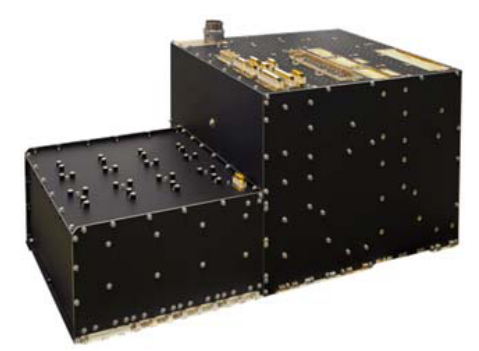

Figure 10. Airbus DS EQM PSCU for T6

The next steps for the PSCU T6 product are the product industrialisation. In the frame of the European GNSS Evolution Program (EGEP), Airbus DS Crisa together with Airbus DS Germany is performing the industrialization of the BEPI PPU Unit. This activity will provide to the market a competitive EP system based on the T6 engine targeting Galileo Second Generation platform as the first customer.

The main goals of the industrialization of the BEPI PPU leading to the EGEP PPU design are therefore:

Minimization of recurrent cost: It is the most important goal for a recurrent production as the required by Galileo mission.

$\checkmark$ Keep the Technology Readiness Level (TRL)

$\checkmark$ System flexibility: As part of the industrialization it has been also considered a goal to get a unit design allowing a greater flexibility at system level, so that with minor (or none) unit adaptations it would be possible to configure different system architectures. 
The EGEP PPU is built of two sub-units:

$\checkmark$ The DANS built off four modules:

- Power Conditioning Module

- LV Control Module

- LV Power Module

- HV Electronics Module

$\checkmark$ The Beam Supply (BS)

One of the major differences is that the unit will not include any type of internal redundancy

The DANS together with the BS and the harness connecting both sub-units built off the complete PPU.

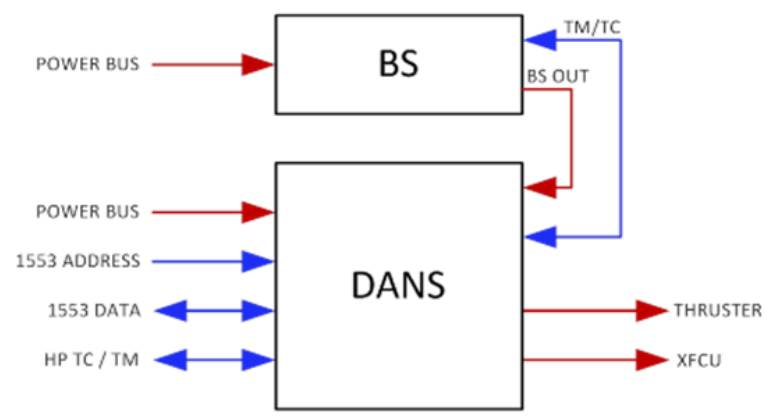

Figure 11 EGEP PPU

This configuration allows expanding the unit to connect two or three thrusters increasing the number of DANS but not BS modules as far as only one of the thrusters is powered at full power or two of them are powered simultaneously with at half power each.

Figure 12 shows the dual thruster configuration.

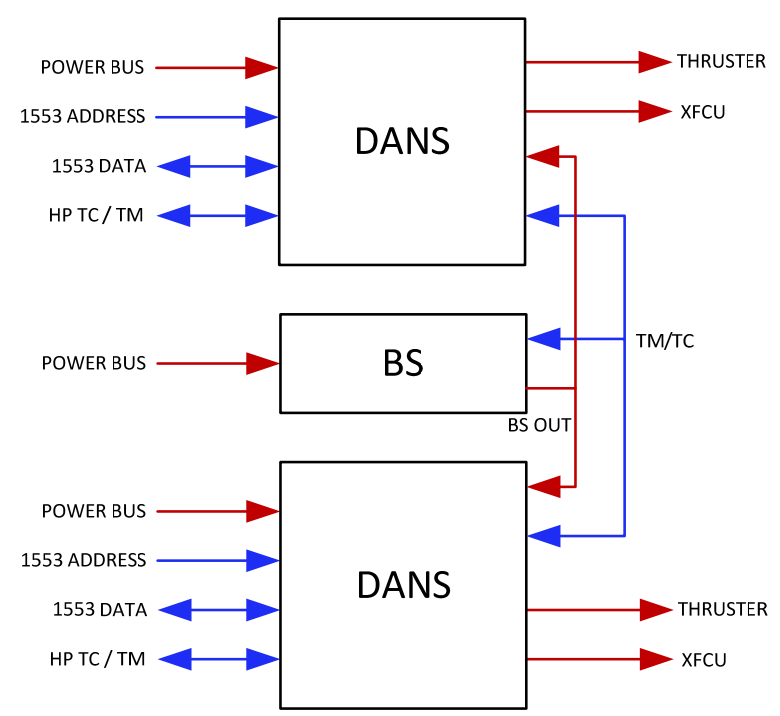

Figure 12 EGEP PPU Two thrusters configuration
The proposed PPU concept also helps to distribute the power dissipated by placing DANS and BS properly separated and allocated to different panels.

After industrialization of the unit the electrical performances expected are the same (or better) than those obtained for BEPI PPU. In particular the expected improvements/design goals expected with respect to former design are:

$\checkmark$ Mass reduction: 29\%

$\checkmark$ Volume reduction: $40 \%$

$\checkmark$ EEE parts reduction: $30 \%$

$\checkmark$ Power Dissipation reduction: $22 \%$

EGEP PPU development includes a design phase and EM model manufacturing and testing. The design phase is finished and EM delivery of the complete PPU for coupling with the thruster is expected in Q2 2017

\subsection{HEMP PPU}

Airbus DS in Germany has developed the PPU for HEMP thruster, the so called PSCU. The PSCU has been developed in the frame of an On-Orbit Demonstration opportunity (SmallGeo). The already qualified hardware is now foreseen for a first flight within the German Heinrich-Hertz (H2) mission.

The Airbus DS PSCU is designed as a modular product (see Figure 13), providing two high voltage power supplies (Anode Supplies) in parallel and operating as main supply for the thrusters, each delivering $1000 \mathrm{~V} /$ 1400W. Redundancy concept approach includes redundancy at PPU level for most of the different power supplies and the capability to drive several thrusters with one single PPU.

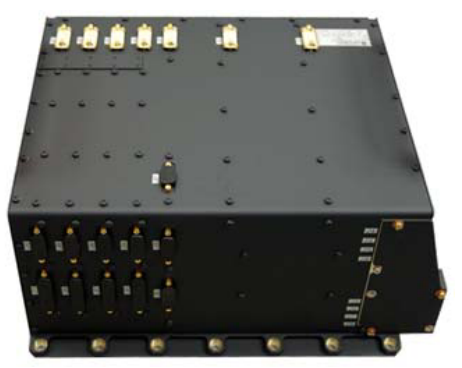

Figure 13 Airbus DS PFM PSCU for HEMP

\subsection{Generic Building Blocks for the PPUs}

The new PPU generation for these technologies will be focused on two main axis:

$\checkmark$ To develop new modular, flexible and cost effective solutions

$\checkmark$ To maximize the communalization or building blocks between the different PPUs for the several technologies. 
For this purpose, Airbus DS Germany is running in the frame of Artes 5.1 a development of generic building blocks to easily customize the PPU to any EP thruster independently of its technology. The maximum communalization will significantly reduce the development efforts, but also the recurrent efforts by applying new technologies and innovative solutions. This activity excludes the High Voltage Power Supply as this already is the main common building block baselined for the different High Voltage PPU designs (see next paragraph).

\section{GENERIC HIGH VOLTAGE POWER SUPPLIES FOR HIGH VOLTAGE THRUSTERS}

Generic High Voltage Power Supply has been developed in Airbus DS Germany and is currently commonly used in all high voltage PPU applications in particular GOCE, AlphaBus, BepiColombo and SmallGEO. The actual generation of modules is used for the Anode Supply Modules for HEMP3050 plasma thruster and the Beam Supply Module (BSM) of the QinetiQ T5 and T6 Ion thruster. Refer to figure 14

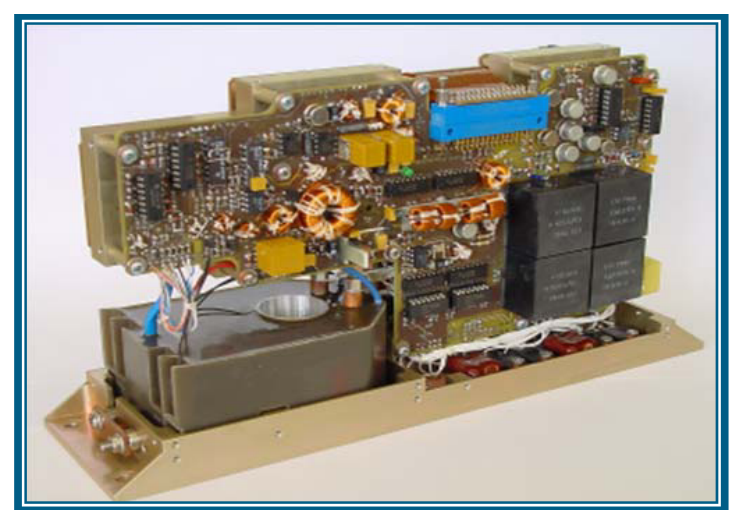

Figure 14 Airbus DS High Voltage Power Supply

The development of a new generation of the generic High Voltage Power Supply has been started in order to meet the needs of Orbit Raising applications. The main technology developments and improvements are:

$\checkmark$ Increase of power from $1,4 \mathrm{~kW}$ up to $5 \mathrm{~kW}$

$\checkmark$ Modular approach $(1,4 / 2,5 / 5 \mathrm{~kW})$

$\checkmark$ Configurable output voltage to optimise the efficiency for orbit raising and station keeping

$\checkmark$ Design for manufacturing / cost

$\checkmark$ Planar transformer \& AMB / direct bonded copper

Generic HVPS qualification is expected to be concluded in 2017 and widely used for the new High Voltage generation PPUs, in particular EGEP PPU as described in the previous chapter

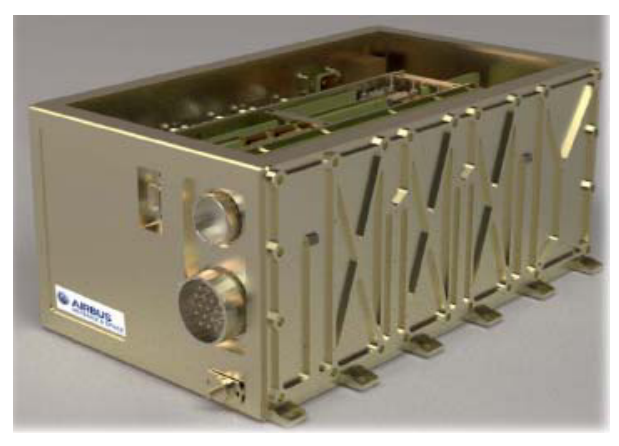

Figure 15 Airbus DS New Generation Generic High Voltage Power Supply

\section{CONCLUSIONS AND OUTLOOK}

This paper has summarized the PPU activities in Airbus DS Space Equipment. The complete portfolio contains solutions for all the Electric Propulsion HET and High Voltage technologies. Airbus DS Space Equipment is continuously improving the designs in order to be able to answer not only to the short term needs, but also the mid and long term needs.

\section{ACKNOWLEDGEMENT}

The authors of this paper wish to thank ESA ESTEC and delegations for their support, in all the relevant activities, summarised as follows:

$\checkmark$ The HKI module as part of the PPU Elektro for HET, described in this paper was developed in the frame of Artes 3/4 program under ESA contract E/0534-01B

$\checkmark$ The PPU industrialisation for T6 has been run under the contract EGEP ID 76.02.02 Industrialization of Power Processing Unit for T6 Electric Propulsion Subsystem

$\checkmark$ DLR Zuwendung "Generic High Voltage Power Supply”, Ref. No. FKZ.: 50 RN 1302

\section{REFERENCES}

[1] IEPC-2015-225/ISTS-2015-b-225 Power Processing Units - Activities in Europe 2015

[2] AIAA 2016-5032 (Propulsion and Energy Forum). Electric propulsion Electronics Activities in Europe 2016

[3] SP2016_ 3125086 (Space propulsion conference 2016). Optimized $5 \mathrm{~kW}$ PPU for Gridded Ion Engine

[4] SP2016 (Space propulsion conference 2016) Power Processing Units activities in Airbus DS Space Equipment. 